\title{
Enhancing the Performance of the Microwave Absorbing Materials by Using Dielectric Resonator Arrays
}

\author{
Omar H. Al-Zoubi ${ }^{1,2}$ and Hameed Naseem ${ }^{2}$ \\ ${ }^{1}$ Department of Renewable Energy Engineering, AL-Albayt University, Mafraq, Jordan \\ ${ }^{2}$ University of Arkansas, Fayetteville, AR 72701, USA \\ Correspondence should be addressed to Omar H. Al-Zoubi; oalzoubi@uark.edu
}

Received 4 November 2016; Revised 3 January 2017; Accepted 9 January 2017; Published 5 April 2017

Academic Editor: Ricardo Perera

Copyright (c) 2017 Omar H. Al-Zoubi and Hameed Naseem. This is an open access article distributed under the Creative Commons Attribution License, which permits unrestricted use, distribution, and reproduction in any medium, provided the original work is properly cited.

\begin{abstract}
We present a technique for enhancing the performance of microwave absorbing materials in terms of weight, thickness, and bandwidth. The introduced technique is based on fabricating the microwave absorbing (MA) material in a structure comprised of an array of circular cylinder dielectric resonators (CDR) backed by a perfect electric conductor (PEC) ground plane. Numerical electromagnetic methods are employed to study the properties of the proposed MA array structures, where 3D full wave simulation using finite-element method is implemented. The obtained results show that the performance of the MA-CDR arrays significantly outperforms that of a flat layer composed of the same material and having equivalent thickness. A flat layer of MA material with thickness of $5 \mathrm{~mm}$ backed by perfect electric conductor (PEC) shows as low as $-50 \mathrm{~dB}$ reflection loss (RL) peak and $\sim 3 \mathrm{GHz} 10-\mathrm{dB}$ bandwidth, whereas an MA-CDR array, composed of the same MA material, of height of $4 \mathrm{~mm}$ can achieve as low as $\sim-50 \mathrm{~dB} R \mathrm{~L}$ peak and $12 \mathrm{GHz} 10$-dB RL bandwidth.
\end{abstract}

\section{Introduction}

The radio frequency and microwave absorbing (MA) materials have numerous applications in various fields such as in military applications (reduction of radar cross section of targets) and communication applications (reduction of electromagnetic interference) [1]. Regardless of the application, weight and thickness reduction and enhancing operating bandwidth are main objectives looked for when designing MA structures. In general, MA materials and structures have to meet general requirements that can be summarized by the following: (i) it should minimize the reflection of EM waves at the air to absorber interface; (ii) it should have strong absorption of electromagnetic waves; (iii) it is expected to have broad bandwidth and angular response; (iv) it should have low weight and thickness [2]. Unfortunately, meeting these competing requirements simultaneously is a challenging task. To reach these objectives and enhance MA materials performance, numerous techniques have been suggested in literature [2]. These techniques can be categorized into two main categories: material and geometry based. In the material based technique, the microwave properties of the material, complex permittivity $(\varepsilon)$ and permeability $(\mu)$, are tailored in a manner to increase electrical and magnetic losses of the material. In the geometry approach, the MA material is constructed in a certain structure with particular geometry and dimensions that reduce the reflection and enhance confinement and absorption of the EM waves inside the structure. Different types of materials can be used as MA material such as carbon, metal and metal particles, conducting polymers, polyaniline conducting polymer, tubules and filaments, and recently composite of nanomaterials [3-9]. Utilizing geometry to create MA structures of high performance is widely used in MA application such as the pyramidal shapes, Dallenbachm and Jaumann layers, honeycomb, and rectangular screen structures $[2,3]$. Frequency selective surfaces and metamaterial structures are other techniques proposed in literature for producing high performance MA structures [5, 6]. Many of these techniques can achieve high performance MA structures. For example, Kim et al. [10] proposed an array of metal-dielectric-multilayer truncated cones structure which showed strong absorption level 
over multibands of operation, with absorption over $90 \%$ in 3.88-6.08 GHz, 9.95-10.46 GHz, and 11.86-13.84 GHz. Other metamaterial MA structures that can achieve strong absorption are presented in [11]. Another example of high performance dielectric MA structure is proposed by Zhao et al. [12]. In that structure, a composite of carbon fibers that are constructed in the form of vertical-arranged carbon fibers and carbon-fiber felt screens can achieve a reflection loss below $-20 \mathrm{~dB}$ in the range of $11.8-18 \mathrm{GHz}$. Despite the fact that these structures have high performance MA properties, they compromise some of the main requirements of the MA materials such as high thickness, narrow band, or complexity of fabrication.

In this paper, regardless of the type of the MA absorbing material, we propose to enhance the already available microwave absorbing materials by only constructing them in the form of CDR arrays. We emphasize employing only the geometry to enhance MA materials performance. The proposed MA structure can simultaneously meet the requirements of low thickness, strong and broad bandwidth absorption, wide angular response, and easiness of fabrication. In this work up to our knowledge, it is the first time to propose using dielectric resonator (DR) for MA applications if arranged in the form of arrays. Up to our knowledge, it is the first time to propose CDR, composed of MA material and arranged in array, to produce high performance MA structure. To this end, this paper is arranged in five sections: Introduction, Theoretical Background, Simulation Strategy, Results and Discussion, and finally the Conclusions. It is worth mentioning that the abstract of this work was presented in the American Advanced Material Congress 2016 [14].

\section{Theoretical Background}

As mentioned in the Introduction, MA materials have to meet general requirements that are difficult to meet simultaneously. Usually, two main objectives are targeted when designing an MA structure: reducing reflection and increasing absorption of EM waves over broadband of frequencies. Reducing the EM wave reflection can be achieved by means of wave impedances matching. Increasing the absorption can be achieved by controlling the material permittivity and permeability (electric and magnetic losses) and by using resonance structures [5]. Broadening the operating bandwidth can be achieved by multilayer structures. In this section we present basic theory of the impedances matching technique for MA material backed by conductive plate and a brief theory about CDR.

2.1. Impedance Matching. In the impedance matching approach, transmission line theory is usually adopted to approximate the propagation of EM waves through air and an MA material. Generally, the RL of an MA layer backed by perfect electrical conductor (PEC) ground plate can be written as [1]

$$
\mathrm{RL}=20 \log |\Gamma|=20 \log \left|\frac{Z_{n}-\eta_{o}}{Z_{n}+\eta_{o}}\right|,
$$

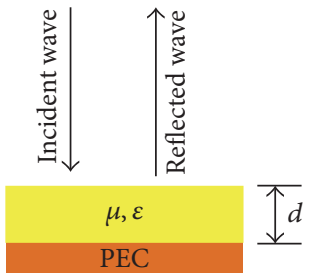

(a) (b)

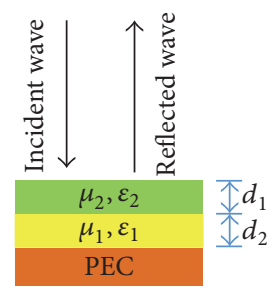

FIgURE 1: MA absorbing material backed by PEC: (a) single MA absorbing materials and (b) double layer MA material for achieving broadband absorption of EM waves.

where $\Gamma$ is the reflection coefficient, $Z_{n}$ is the wave impedance in the MA material, and $\eta_{o}$ is the wave impedance in air. For the configuration of a single flat layer of homogenous MA material backed by PEC, RL can be written as [1]

$$
\begin{aligned}
\mathrm{RL} & =20 \log |\Gamma| \\
& =20 \log \left|\frac{\sqrt{\mu / \varepsilon} \tanh (j(2 \pi f d / c) \sqrt{\mu \varepsilon})-1}{\sqrt{\mu / \varepsilon} \tanh (j(2 \pi f d / c) \sqrt{\mu \varepsilon})+1}\right| .
\end{aligned}
$$

In this configuration, the RL and the microwave absorbing properties depend on the MA material thickness, $d$, and electrical properties of the MA material, $\mu$ and $\varepsilon$. To enhance the RL of the flat layer configuration, graded interface can be utilized, which can be achieved by gradually loading the MA material to produce gradual wave impedance change. Pyramidal shape structures are also used to achieve this gradual wave impedance change. Both techniques can be used together to achieve better impedance matching and high MA performance as in anechoic chambers [15]. Stacked layers are another approach for enhancing impedance matching and RL bandwidth of MA structures [2]. Figure 1 shows a schematics of single and double flat MA layers backed by PEC ground plane. In such flat configurations, the microwave absorbing properties can be modulated by manipulating the layer thicknesses, which can be inferred from (2) for single layer.

2.2. Dielectric Resonators. In fact, arrays of dielectric circular cylinders are widely proposed and studied in the realm of optics for enhancing optical properties of materials [16]. Light is absorbed in materials by mechanisms that are different than that of the microwave. Despite this, the principle of using CDR array is utilized in this work to enhance the MA materials performance. Dielectric resonators (DR) are widely used as antennas in communication applications [17]. As antenna, DR is usually composed of a dielectric martial of low microwave and RF losses and high relative permittivity. In the contrary to the DR antenna, in this work we implement materials with high electrical and magnetic losses to construct CDR array that behave as MA absorbing structure. In this approach, the incident EM wave couples with the CDR at multiple resonance frequencies (modes), where the EM fields are partially confined and absorbed inside the CDR. Different modes can be excited inside a CDR that depends 
on its geometry and dimensions. For a single CDR on top of PEC ground plane the following approximation can be used to find resonance modes [18]:

$$
\left(\begin{array}{c}
f_{\mathrm{TM}_{n p m}} \\
f_{\mathrm{TE}_{n p m}}
\end{array}\right)=\frac{c}{2 \pi r \sqrt{\varepsilon \mu}} \sqrt{\left(\begin{array}{l}
X_{n p}^{\prime} \\
X_{n p}
\end{array}\right)^{2}+\left(\frac{(2 m+1) \pi a}{2 h}\right)^{2}},
$$

where $f_{\mathrm{TM}_{n p m}}$ is the $\mathrm{TM}_{n p m}$ mode frequency, $f_{\mathrm{TE}_{n p m}}$ is the $\mathrm{TE}_{n p m}$ mode frequency, $X_{n p}^{\prime}$ is the $p$ th root of the $n$th order Bessel function derivative, $X_{n p}$ is the $p$ th root of the $n$th order Bessel function, $r$ is the radius, and $h$ is the high of the CDR, respectively. Such approximation is valid with reasonable accuracy for single CDR within range of physical dimensions [18]. In the CDR arrays, this approximation can be used in the case of diluted CDR arrays. For the case of dense arrays, where mutual coupling between the CDR is significant, coupling modes start to be excited [19], and the approximation given in (3) is not quite valid for predicting the different resonance modes that can appear in the array structure. Nevertheless, our focus in this work is to prove the concept of enhancing MA material performance, by forming the MA material in the form of CDR array structure, by using numerical methods. However, the CDR approximation equation can be used to predict the resonance modes and the range of operating frequencies of MA-CDR arrays.

\section{Simulation Strategy}

There are several techniques that can be used to solve Maxwell's equations numerically such as finite-difference time domain (FDTD) method, transfer-matrix method (TMM), method of moment (MoM), and finite-element method (FEM). Numerous commercial software packages can be used to calculate properties of MA materials and structures, such as ANSYS HFSS (FEM based, frequency domain), CST Microwave Studio (FDTD based, time domain), and FEKO (MoM based). In this work, ANSYS HFSS simulation package is selected to study the EM wave interaction with the MA-CDR array structures and to calculate their properties. HFSS software offers the capability of simulating complex structures with subwavelength dimensions and the capability to implement the exact dispersive microwave properties of materials in the simulations. All of the simulations in this work are performed on PC equipped with Intel(R) Core(TM) i7-4790 CPU @ 3.6 GHz and 16 GB RAM.

3.1. CDR Array Modeling. The MA-CDR array is modeled by infinite 2D array in the $X$ and $Y$ directions and composed of identical circular cylinders that are arranged in square lattice structure, as Figure 2(a) shows. The circular cylinders are composed of an MA material with known microwave properties taken from [13]. This material is a composite material that is loaded with a nanopowder material, Fe20Ni8, that has relative permittivity in the range of $\sim 16-\sim 28$ and relative permeability in the range of $\sim 1-\sim 1.8$. In this work, we apply the proposed technique on this composite MA material to further enhance its performance, which is the main objective of this study. It is worth mentioning that the selected material is dispersive; therefore its frequencydependent microwave properties are implemented in the simulations by using lookup tables. To realize the infinite $2 \mathrm{D}$ array in the simulation domain, we implement a unit cell with periodic boundary conditions in the $X$ and $Y$ directions. Perfect magnetic walls (PMW) and perfect electric conductor (PEC) walls are imposed to the simulation domain of the unit cell as shown in Figure 2. The dimensions of the array and the CDR are illustrated in Figure 2, where identical CDR elements are arranged in square lattice structure with period (lattice constant) that is assigned $W$ letter. In this work, normal plane wave incidence on the array is assumed, as demonstrated in Figure 2(a).

3.2. Scattering Parameters. The performance of the proposed MA-CDR arrays is quantified by the bandwidth of RL spectrum and the absorbance of the arrays calculated as $A(\omega)=1-T(\omega)-R(\omega)$, where $T(\omega)$ is the transmittance and $R(\omega)$ is the reflectance. The scatter parameters are used to calculate $T(\omega)$ and $R(\omega)$, where $R(\omega)=\left|S_{11}(\omega)\right|^{2}$ and $T(\omega)=\left|S_{12}(\omega)\right|^{2}$. Due to the fact that the structure is backed by conductive ground plane, the transmittance, $T(\omega)$, equals zero and the absorbance reduces to $A(\omega)=1-R(\omega)$.

3.3. Discretization of the Simulation Domain. In the FEM, the simulation domain is discretized into subelements. The accuracy of Maxwell equations solutions depends on the discretization resolution of the simulation domain. Theoretically, the discretization can be set as fine as desired. However, extremely fine resolution of the discretization requires large computation resources, in terms of RAM and time, that becomes prohibitively large. In order obtain reasonable accuracy of the simulation results, using reasonable computation resources, the domain discretization resolution should be set to certain value. For this purpose, we run convergence analysis of the solution at different discretization resolutions. HFSS software uses adaptive meshing algorithm to discretize the 3D simulation domain into tetrahedral subelements. The maximum length of the tetrahedral edges can be set not to exceed certain value, which determines the discretization resolution. For the proposed array, we conducted convergence analysis of the solution at different discretization step sizes. In this analysis, the maximum length of the tetrahedral edges is set to be $0.1 \mathrm{~mm}$ up to $1.0 \mathrm{~mm}$, with $0.1 \mathrm{~mm}$ increment each run. The results in Figure 3(a) show the RL spectrum of an array of $2.2 \mathrm{~mm}$ radius, $5.0 \mathrm{~mm}$ height, and 5.7 lattice constant, calculated at different discretization resolutions. Figure 3(b) shows zoomed part of the RL spectrum, where the spectrum lines are corresponded to the discretization level shown in Figure 3(c). The top view of a CDR of $2.2 \mathrm{~mm}$ radius at different discretization step sizes is illustrated in Figure 3(c). One can see that the solution converges to the resonance frequency at $0.5 \mathrm{~mm}$ discretization step size, which is $0.25 \%$ of the CDR radius. However, to guarantee the accuracy of the results of different dimension arrays, we set the maximum discretization step to be $10 \%$ of the CDR radius in all of the simulations in this work. 


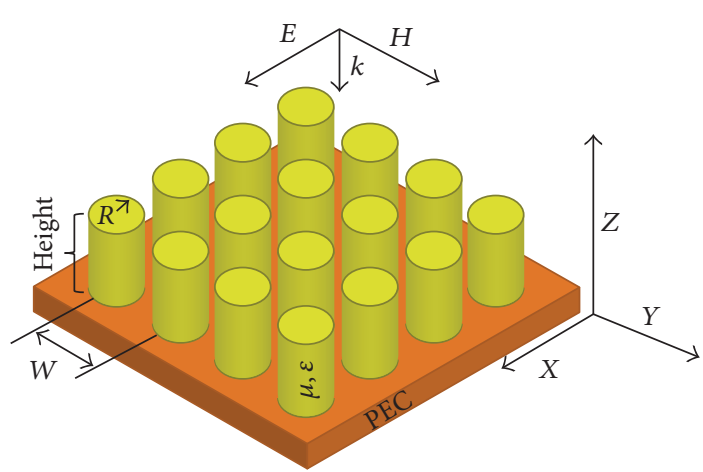

(a)

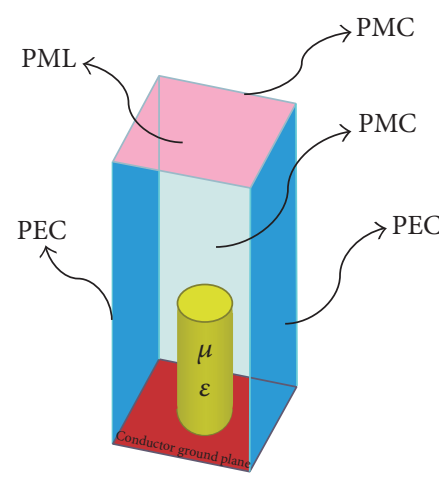

(b)

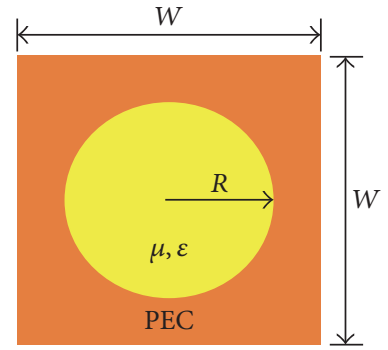

(c)

FIGURE 2: Schematic shows the CDR array on top of PEC ground plane in (a), unit cell used to simulate the array in the simulation domain in (b), and top view of the unit cell showing the dimensions of the CDR and the unit cell with lattice constant $(W)$ in $(c)$.

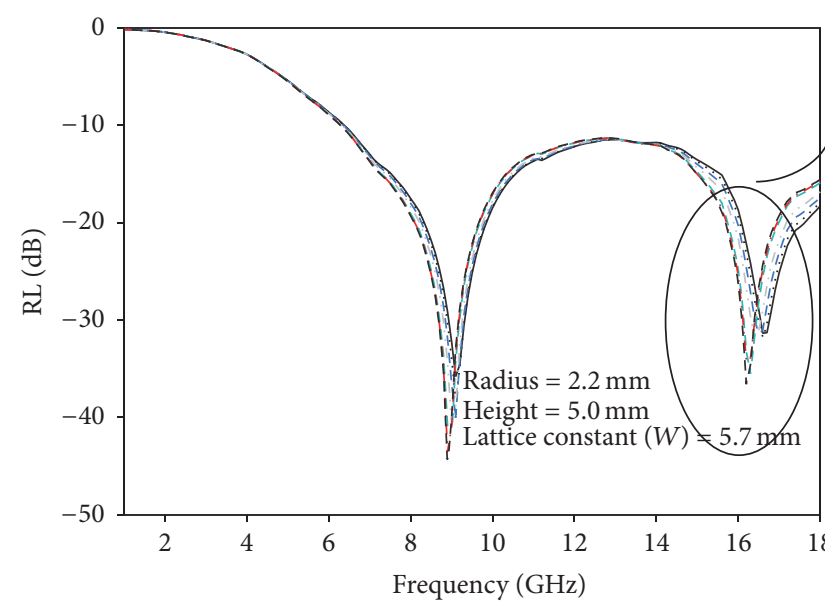

(a)

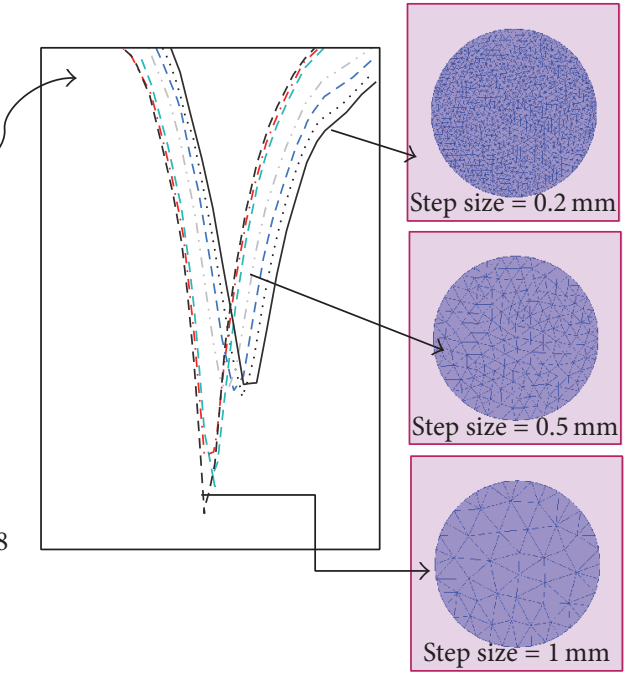

(b)

(c)

FIGURE 3: The RL spectrum of an MA-CDR array calculated at different discretization step sizes (a), zoomed part of the RL spectrum (b), and top view of the CDR including the discretization mesh at different levels in (c).

3.4. Validation of the Simulation. Due to the lack of microwave measurement devices in the current time and in order to validate the simulation results, we conducted simulation of different published works and compare the results of our simulation with them. In the following context we show one of these published results compared with our simulation results. The setup of that published work is in the form of a MA layer backed by a PEC ground plane as shown in the inset of Figure 4, taken from [13]. Good agreement between the results of our simulation and those from [13] is obtained, as shown in Figure 3. This comparison shows that the simulation setup used in this work and the obtained results are quite accurate and realistic.

3.5. Parametric Analysis. The proposed MA-CDR array design has three degrees of freedom: radius and height of the CDR and the lattice constant of the array. These parameters are key elements in determining the absorption strength and
RL spectrum broadness, as will be shown in Section 4. To determine the effect of the different parameters of the DR array on its performance as an MA, we adopted parametric analyses. We choose to fix the value of two parameters at a time and sweep the other value, to investigate the effect of each parameter in isolation from others. For the current material under study, the ranges of parameters are swept as 1 to $5 \mathrm{~mm}$ for radius values with $1 \mathrm{~mm}$ increment step, 1 to $14 \mathrm{~mm}$ for the thickness values with $1 \mathrm{~mm}$ increment step, and 5 to $7 \mathrm{~mm}$ for the lattice constant values with $0.1 \mathrm{~mm}$ increment step. The RL is calculated over the frequency range of $1-18 \mathrm{GHz}$ with $0.1 \mathrm{GHz}$ increment step.

\section{Results and Discussion}

As mentioned earlier, the microwave absorbing properties of the flat MA layer backed by PEC can be modulated simply by manipulating its thickness. In an MA-CDR array, there are 


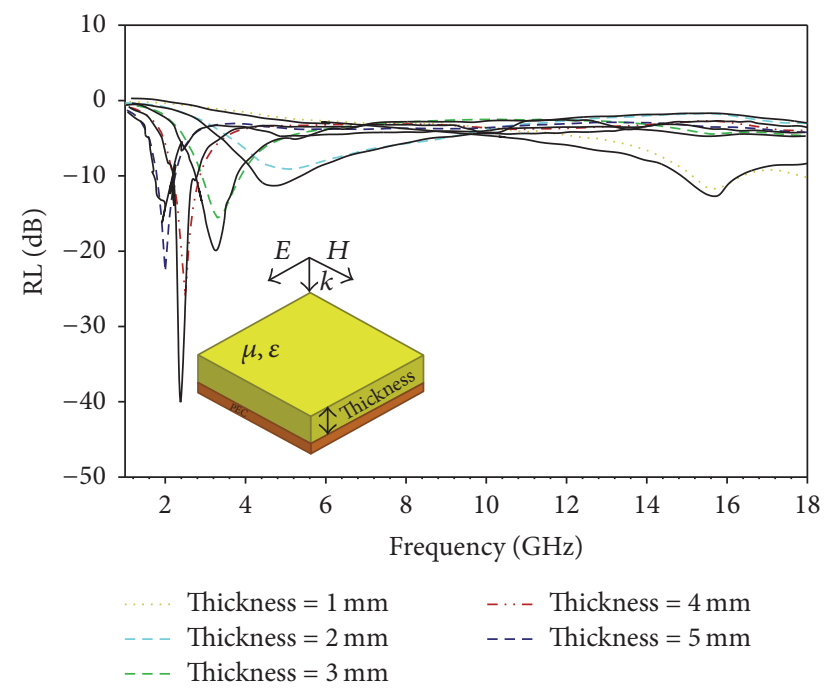

FIgURE 4: The reflection loss spectra of different thicknesses layers of Fe20Ni8 nanopowders composite MA material backed by PEC ground plane: results of [13] (solid black) and our simulation results (colored doted) [14].

three different parameters that affect its microwave absorbing properties. These parameters are the CDR radius, height, and the lattice constant of the array. Each of these parameters has noticeable effect on the RL peaks position and value, which eventually affects the performance of the MA absorption array. The parameters offer three degrees of freedom for controlling and tuning the RL spectrum of an MA-CDR array. In the following subsections we introduce the effect of each parameter on absorption strength and bandwidth. Optimal performance of the arrays, in the sense of RL spectrum bandwidth and level, is obtained through parametric analysis as mentioned in Section 3.

4.1. Height Effect. To study the effect of the CDR height on the array performance in isolation from other parameters, the lattice constant of the array and radius of the CDR are fixed to certain values, $W=15 \mathrm{~mm}$ and $R=5 \mathrm{~mm}$. Figure 5 shows that the RL peaks shift to higher frequencies as the CDR height increases. This behavior can be related to the resonance modes of the CDR, which can be inferred from (3). For the given lattice constant and CDR radius values, one can see that the RL has optimal value and bandwidth at certain CDR height, and it is not necessary that as the height increases the RL enhances. RL value as low as $-50 \mathrm{~dB}$ appears at $\sim 5 \mathrm{GHz}$ for the MA-CDR array of $13 \mathrm{~mm}$ height with 10 - $\mathrm{dB}$ $\sim 4 \mathrm{GHz}$ bandwidth and $\sim 2 \mathrm{GHz} 20-\mathrm{dB}$ bandwidth. Another absorption peak can be seen at $\sim 16 \mathrm{GH}$ where the 10 -dB bandwidth is $\sim 3 \mathrm{GHz}(\sim 15-18 \mathrm{GHz})$ and the 20 -dB bandwidth is $\sim 1 \mathrm{GHz}$. The absorption level of the array, in the case of the $13 \mathrm{~mm}$ height of the CDR, exceeds $90 \%$ in the ranges of $\sim 3 \mathrm{GHz}$ to $\sim 7 \mathrm{GHz}$ and $\sim 15 \mathrm{GHz}$ to $18 \mathrm{GHz}$. For the ranges of $\sim 4 \mathrm{GHz}$ to $6 \mathrm{GHz}$ and $\sim 15$ to $17 \mathrm{GHz}$, the absorption level exceeds $99 \%$. It can be seen that the height can control the RL peak position, value, and bandwidth of the RL. For this reason, the height of the CDR in an array can be utilized to

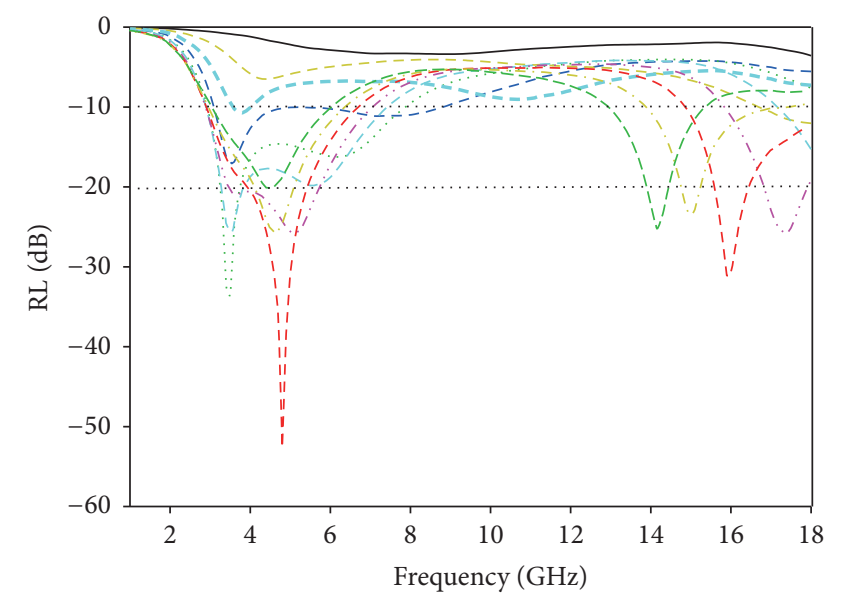

$$
\begin{aligned}
- \text { Height } & =2 \mathrm{~mm} \\
--- \text { Height } & =4 \mathrm{~mm} \\
--- \text { Height } & =6 \mathrm{~mm} \\
--- \text { Height } & =8 \mathrm{~mm} \\
\ldots . . \text { Height } & =10 \mathrm{~mm}
\end{aligned}
$$

FIgURE 5: Reflection loss spectra of the MA-CDR array at different heights and with fixed lattice constant of $15 \mathrm{~mm}$ and radius of $5 \mathrm{~mm}$.

tune the operating frequency range of the MA-CDR arrays, especially when there are no restrictions on the height of the array.

4.2. Radius Effect. The radius of a single CDR is a key element in determining its resonance frequencies, which applies to MA-CDR arrays. Equation (3) shows the dependency of the resonance mode frequencies on the radius value of the CDR. In the MA-CDR arrays, this dependency is still valid, but the single CDR approximation, given in (3), is not quite valid for determining the resonance frequencies values of an array. To study the effect of the radius value of the CDR in the overall performance of the MA-CDR array, we conducted simulation with fixed lattice constant and height of the array. Figure 6 shows the effect of increasing the radius of the CDR of an array with height of $5 \mathrm{~mm}$ and lattice constant of $6.6 \mathrm{~mm}$. The RL peak shifts to a higher frequency as the radius decreases. This trend can be predicted from the CDR approximation in (3). However, the values of the peaks of the RL spectrum are not only governed by the radius and the height of the MA-CDR, but also the mutual coupling between the CDR in the array. The two peaks that occur in the range of $1-18 \mathrm{GHz}$ can be attributed to the resonance modes of the CDR. As Figure 6 shows, the radius has significant impact on the fundamental mode position, whereas it has slight influence on the upper mode. In the case of lattice constant of $6.6 \mathrm{~mm}$ and CDR radius of $2.2 \mathrm{~mm}$, the $20-\mathrm{dB}$ RL bandwidth is $\sim 5 \mathrm{GHz}$ ( $\sim 9 \mathrm{GHz}$ to $\sim 14 \mathrm{GHz}$ ), which means $99 \%$ absorption of the incident EM waves, as shown in Figure 5.

4.3. Array Lattice Constant Effect. In the previous subsection, the height and the radius of CDR in an MA-CDR array were demonstrated, where $\mathrm{CDR}$ approximation equation can be used to predict the effect of these two parameters. In 


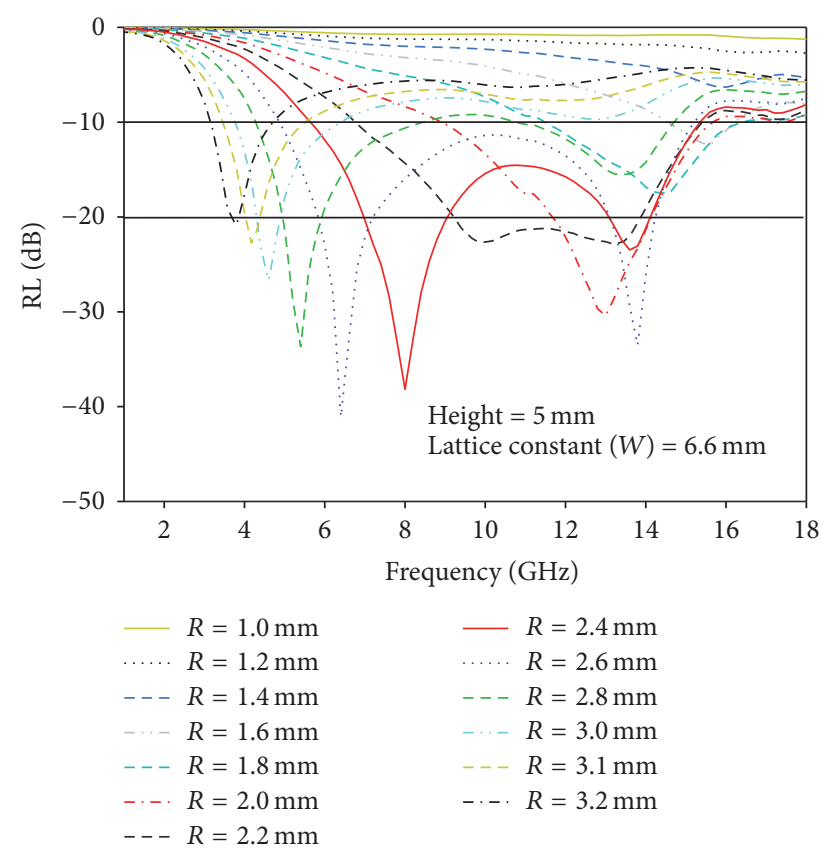

FIgURE 6: Reflection loss spectra of the MA-CDR array that has fixed lattice constant $(W)$ of $6.6 \mathrm{~mm}$ and height of $5 \mathrm{~mm}$.

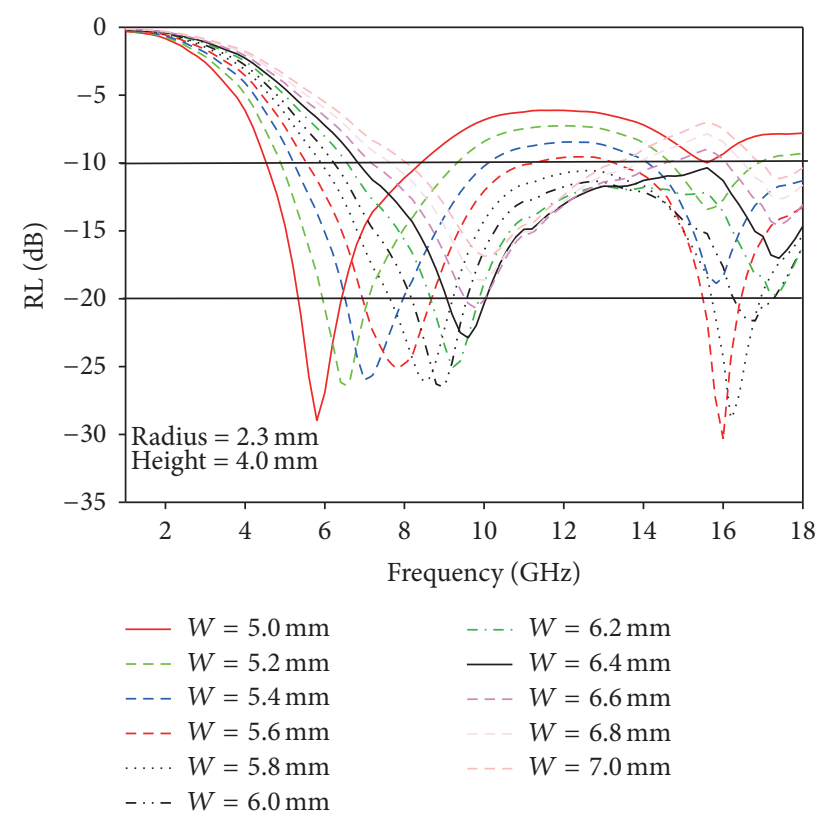

FIgURE 7: Reflection loss spectra of the MA-CDR array that has fixed CDR radius $(R)$ of $2.3 \mathrm{~mm}$ and height of $4.0 \mathrm{~mm}$.

this subsection, the lattice constant of the MA-CDR array is introduced. One can look at the lattice constant as the gap distance between the CDR [17]. This distance plays important role in the mutual coupling between the adjacent CDR in the array. It can be seen from Figure 7 that the peak position of the RL is highly dependent on the lattice constant. In this figure, the lattice constant value is changed incrementally between 5 and $7 \mathrm{~mm}$ for an array of $4 \mathrm{~mm}$ height and $2.3 \mathrm{~mm}$
$\mathrm{CDR}$ radius. The lattice constant value has noticeable effect on the RL spectrum level, operating frequencies, and the RL bandwidth of the MA-CDR array. One can see that RL spectrum of 10 -dB bandwidth of $\sim 10 \mathrm{GHz}$ (from $\sim 8$ to $18 \mathrm{GHz}$ ) is achieved at the lattice constant value of $5.8 \mathrm{~mm}$, as Figure 7 shows.

4.4. The Optimal Performance of MA-CDR Array. In the previous subsections, to demonstrate the effect of array dimensions on the MA-CDR performance, the RL spectra of the MA-CDR arrays at certain dimension values are shown. It is obvious that the performance of the array is dependent on the combination of its physical dimension values. To obtain the optimal performance, in the sense of absorption level and RL bandwidth, optimization of the three parameters needs to be conducted. We adopted parametric analysis in this work in order to find the optimal performance of the array and to explore the potentials of the array performance. We selected the 10- $\mathrm{dB}$ bandwidth as the criterion for optimal performance. Figures 8(a) and 8(c) show two optimal RL spectra for the MA-CDR array at two different combinations of height, radius, and lattice constant values. For $5 \mathrm{~mm}$ height and $7 \mathrm{~mm}$ lattice constant, a $10-\mathrm{dB}$ bandwidth as high as $10.2 \mathrm{GHz}$ ( 4.8 to $15 \mathrm{GHz}$ ) is achieved when the radius is $2.7 \mathrm{~mm}$. In this range band, the absorption exceeds $90 \%$ as Figure 8(c) shows. A more enhanced array performance, in the sense of bandwidth and absorption level, can be achieved by reducing the height of the array and lattice constant as Figure 8(b) shows. It can be shown that the bandwidth and the absorption level are enhanced, where $12.2 \mathrm{GHz} 10$ $\mathrm{dB}$ bandwidth is achieved when the radius is $2.3 \mathrm{~mm}$. It is worth mentioning that $99 \%$ absorption level can also be achieved in the ranges of $7-9.6 \mathrm{GHz}$ and $15.2-18.0 \mathrm{GHz}$ as Figure $8(\mathrm{~d})$ demonstrates. In fact, Figure 8 shows how the operating frequency, bandwidth, and the absorption level can be manipulated by the parameter values of the array. An important observation is that the operating frequency is extended beyond the $18 \mathrm{GHz}$ limit shown in this work, as Figure 8(a) suggests.

Comparison of the performance of the MA-CDR array with that of the flat layer, Figure 4, shows the significantly enhanced performance of the MA-CDR arrays.

\section{Conclusions}

We introduced a new technique to enhance the performance of MA materials. The technique is based on geometry, where an MA material is fabricated in the form of DR that are arranged in arrays. The performance of the proposed MACDR arrays of different dimensions has been studied. Strong and broadband microwave absorption can be achieved by using low thickness MA-CDR arrays. It is found that the MACDR arrays backed by a conductor, compared with the same thickness bulk of counterpart material, have significantly enhanced MA performance. One finding in this work is that the performance of an MA-CDR array is highly dependent on three parameters, which give the designer three degrees of freedom. That is, the RL spectrum of a certain MA-CDR 


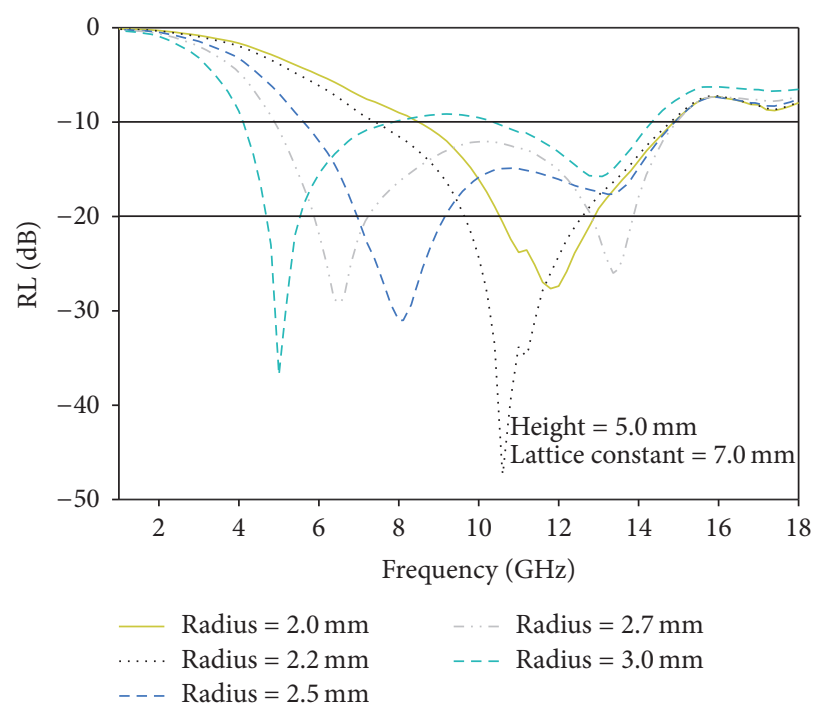

(a)

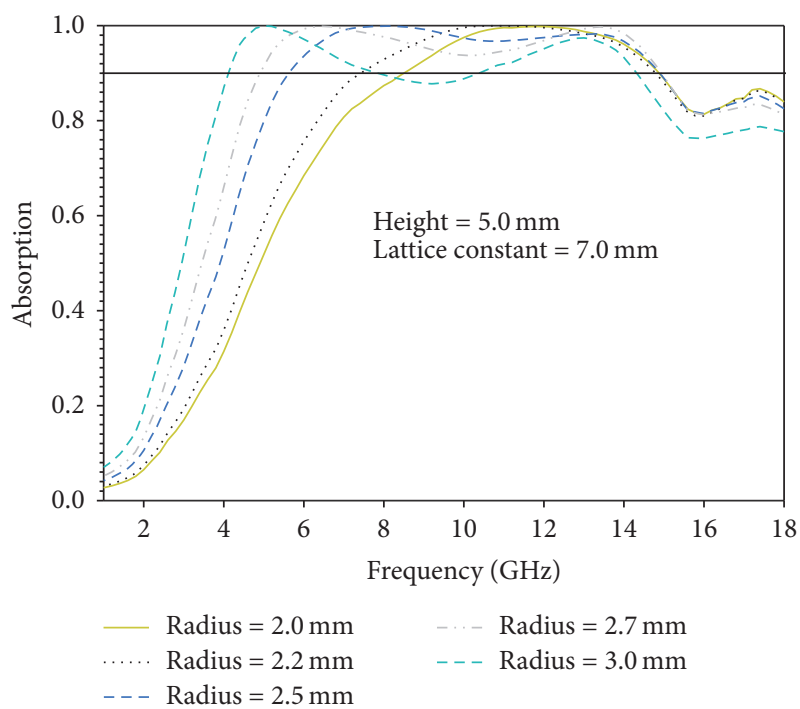

(c)

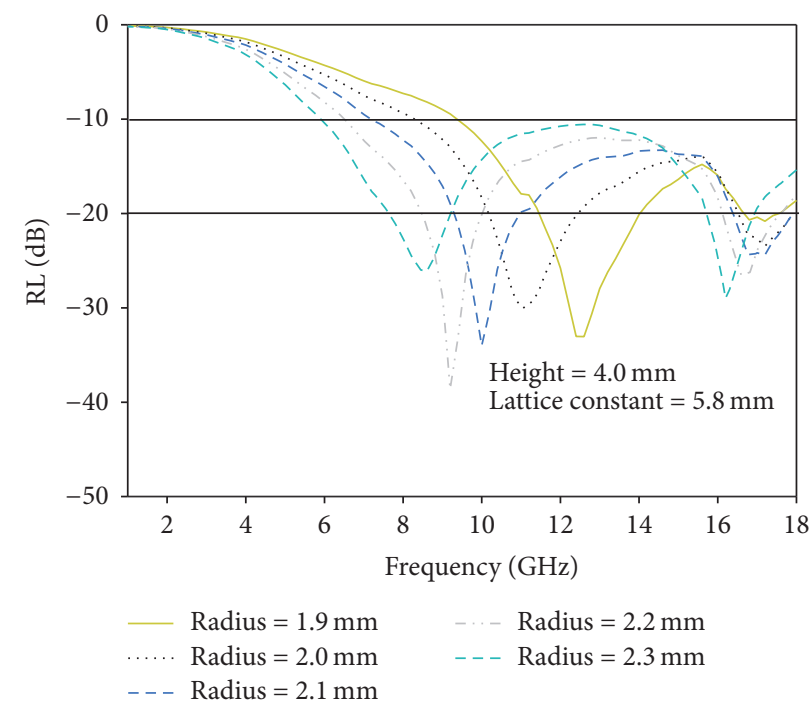

(b)

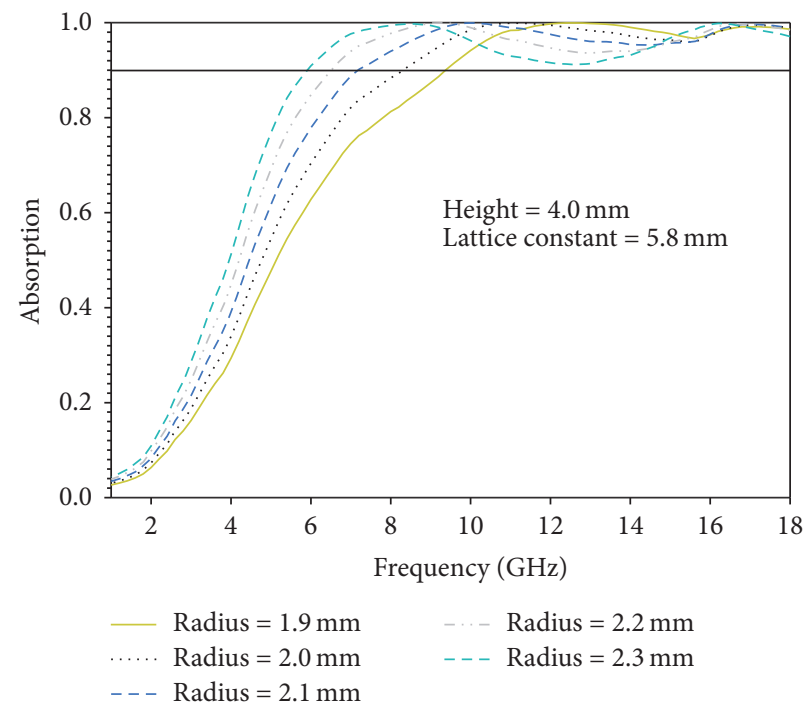

(d)

FIGURE 8: Reflection loss and absorption spectra of infinite MA-CDR array of $5 \mathrm{~mm}$ height and $7 \mathrm{~mm}$ lattice constant in (a) and (c) and of $4 \mathrm{~mm}$ height and 5.8 lattice constant in (b) and (d).

layer can be tuned and tailored by these three parameters of the array dimensions. The obtained results suggest that ultra-thin MA structures with broadband RL can be achieved by using the CDR array technique. Such findings can pave the road for producing new generation of high performance low profile MA layers. It is worth mentioning that the proposed technique can further enhance already available high performance MA structures, such as pyramidal MA structure, by texturing their surfaces in the form of CDR array.

\section{Conflicts of Interest}

The authors declare that there are no conflicts of interest regarding the publication of this paper.

\section{References}

[1] F. Qin and C. Brosseau, "A review and analysis of microwave absorption in polymer composites filled with carbonaceous particles," Journal of Applied Physics, vol. 111, no. 6, Article ID 061301, 2012.

[2] P. Saville, Review of Radar Absorbing Materials, Defence Research \& Development Canada, 2005.

[3] G. J. H. Melvin, Q.-Q. Ni, Y. Suzuki, and T. Natsuki, "Microwaveabsorbing properties of silver nanoparticle/carbon nanotube hybrid nanocomposites," Journal of Materials Science, vol. 49, no. 14, pp. 5199-5207, 2014.

[4] J. Grandidier, R. A. Weitekamp, M. G. Deceglie et al., "Solar cell efficiency enhancement via light trapping in printable resonant dielectric nanosphere arrays," Physica Status Solidi (A), vol. 210, no. 2, pp. 255-260, 2013. 
[5] A. Munir, "Microwave radar absorbing properties of multiw alled carbon nanotubes polymer composites: a review," Advances in Polymer Technology, 2015.

[6] B. D. Che, B. Q. Nguyen, L.-T. T. Nguyen et al., "The impact of different multi-walled carbon nanotubes on the X-band microwave absorption of their epoxy nanocomposites," Chemistry Central Journal, vol. 9, no. 1, article 10, 2015.

[7] P. Savi, M. Miscuglio, M. Giorcelli, and A. Tagliaferro, "Analysis of microwave absorbing properties of epoxy MWCNT composites," Progress in Electromagnetics Research Letters, vol. 44, pp. 63-69, 2014.

[8] L. Olmedo, P. Hourquebie, and F. Jousse, "Microwave absorbing materials based on conducting polymers," Advanced Materials, vol. 5, no. 5, pp. 373-377, 1993.

[9] T. McNally and P. Pötschke, Polymer-Carbon Nanotube Composites: Preparation, Properties and Applications, Elsevier, 2011.

[10] Y. J. Kim, Y. J. Yoo, K. W. Kim, J. Y. Rhee, Y. H. Kim, and Y. Lee, "Dual broadband metamaterial absorber," Optics Express, vol. 23, no. 4, pp. 3861-3868, 2015.

[11] Y. P. Lee, J. Y. Rhee, Y. J. Yoo, and K. W. Kim, Metamaterials for Perfect Absorption, vol. 236, Springer, 2016.

[12] N. Zhao, T. Zou, C. Shi, J. Li, and W. Guo, "Microwave absorbing properties of activated carbon-fiber felt screens (verticalarranged carbon fibers)/epoxy resin composites," Materials Science and Engineering B: Solid-State Materials for Advanced Technology, vol. 127, no. 2-3, pp. 207-211, 2006.

[13] H. Zhao, Z. Zhu, C. Xiong, X. Zheng, and Q. Lin, “The influence of different $\mathrm{Ni}$ contents on the radar absorbing properties of FeNi nano powders," RSC Advances, vol. 6, no. 20, pp. 1641316418, 2016.

[14] IAAM, Proceedings and Abstracts Book, Proceeding-bookAAMC-2016-USA, 2016, http://www.vbripress.com/aamc.

[15] X. C. Tong, Advanced Materials and Design for Electromagnetic Interference Shielding, CRC Press, 2008.

[16] O. H. AL-Zoubi, T. M. Said, M. A. Alher, S. EL-Ghazaly, and H. Naseem, "Broadband high efficiency silicon nanowire arrays with radial diversity within diamond-like geometrical distribution for photovoltaic applications," Optics Express, vol. 23, no. 15, pp. A767-A778, 2015.

[17] A. Trubin, Lattices of Dielectric Resonators, Springer, 2015.

[18] C. A. Balanis, Advanced Engineering Electromagnetics, John Wiley \& Sons, 2012.

[19] P. N. Fletcher, M. Dean, and A. R. Nix, "Mutual coupling in multi-element array antennas and its influence on MIMO channel capacity," Electronics Letters, vol. 39, no. 4, pp. 342-344, 2003. 


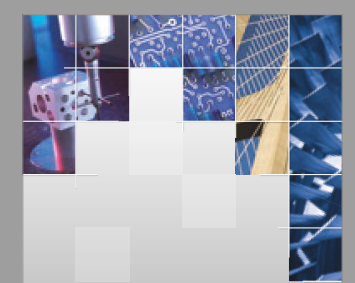

\section{Enfincering}
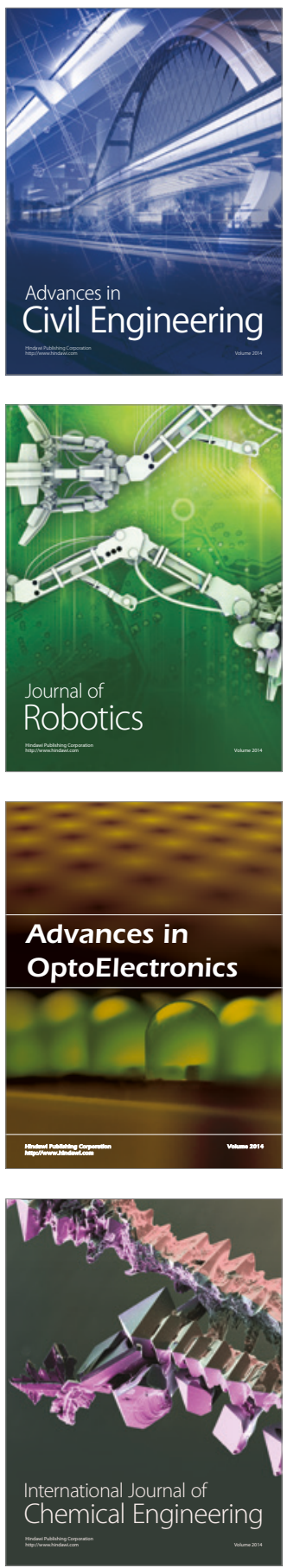

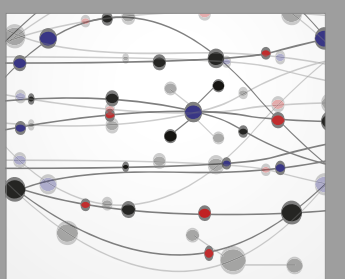

The Scientific World Journal

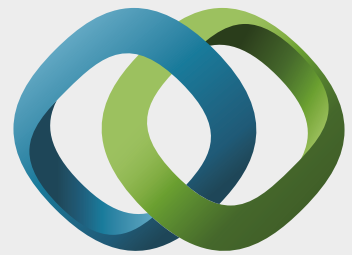

\section{Hindawi}

Submit your manuscripts at

https://www.hindawi.com
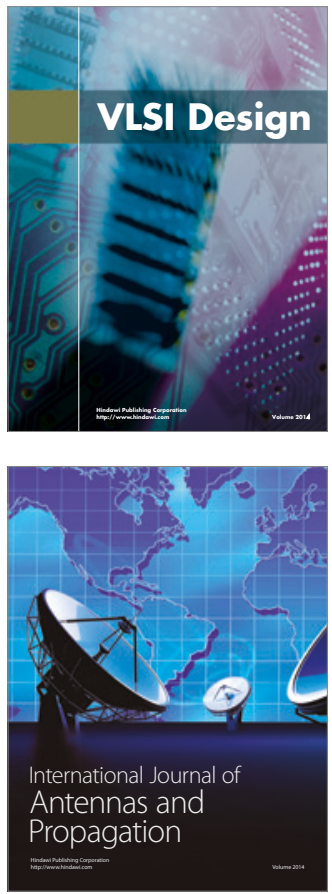

\section{Rotating}

Machinery
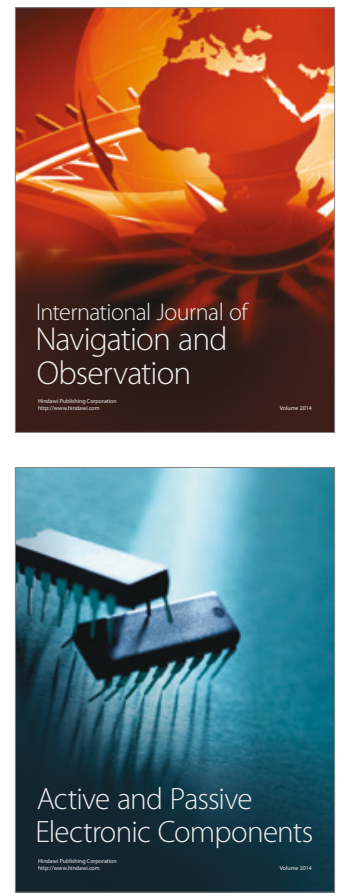
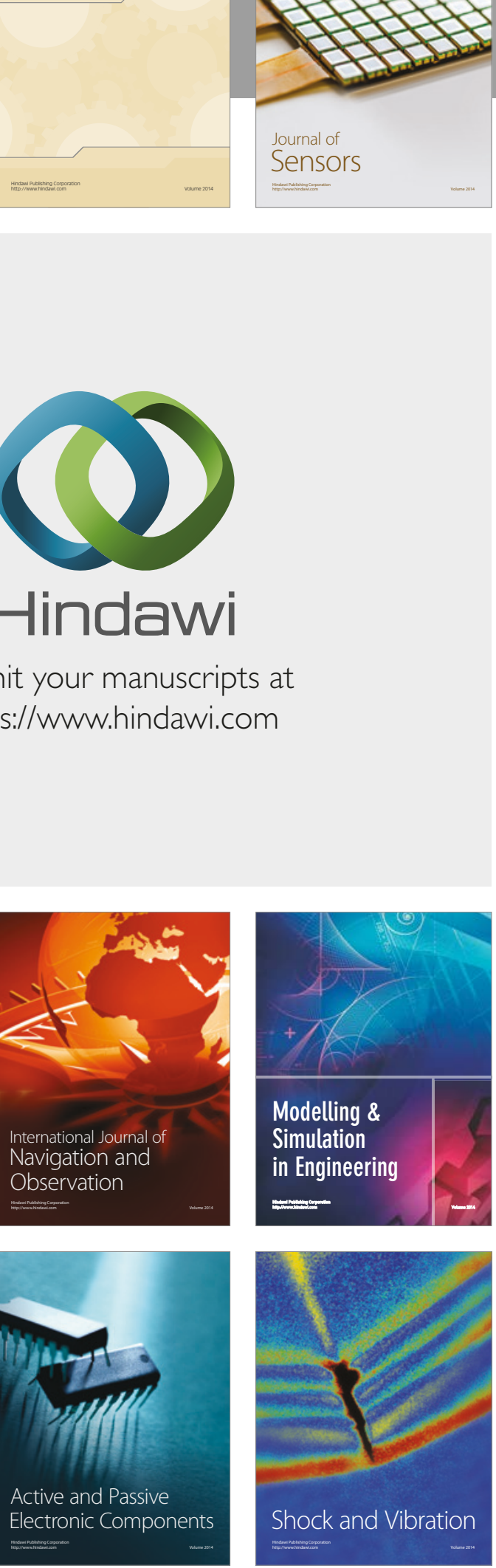
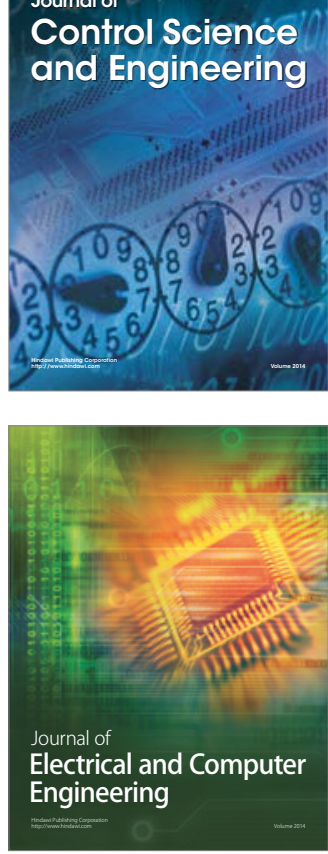

Distributed

Journal of

Control Science

and Engineering
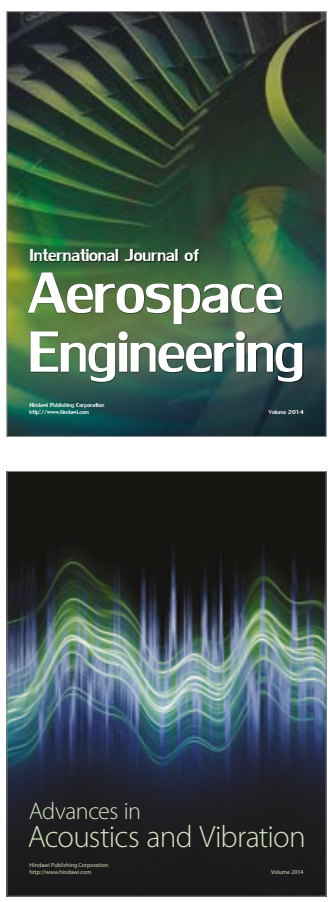

Sensor Networks 